\title{
Engineering
}

\section{Review on Citrinin: Production, Effect of Some Plant Extracts and Gene Involved in its Biosynthesis}

\section{Alaa Mostafa Abou-Zeid \\ Botany Department, Faculty of Science, Tanta University, Tanta, Egypt}

\section{Introduction}

Citrinin is the one of the well-known mycotoxins, which is possibly spread all over the world. It is originally isolated from Penicillium citrinum and now produced by a variety of other fungi. Citrinin was produced in liquid potato-dextrose medium and determined by TLC and HPLC. Extracts of some plants reduce or inhibit citrinin production. Some of the genes involved in Citrinin biosynthesis were discussed.

\section{Physicochemical Properties}

Citrinin $\left[\mathrm{C}_{13} \mathrm{H}_{14} \mathrm{O}_{5}\right.$, IUPAC: (3R, 4S)-4, 6-dihydro-8-hydroxy-3, 4, 5-trimethyl-6-oxo-3H-2-benzopyran-7-carboxylic acid; CAS No.: 51875-2] (Figure 1), is an acidic lemon-yellow crystal with maximal UV absorption at $250 \mathrm{~nm}$ and $333 \mathrm{~nm}$ (in methanol), melting at $172^{\circ} \mathrm{C}$ [1]. It is sparingly soluble in water but soluble in dilute sodium hydroxide, sodium carbonate, or sodium acetate; in methanol, acetonitrile, ethanol, and most of other polar organic solvents [2].

It is a mycotoxin originally isolated from Penicillium citrinum. It has been found to be produced by a variety of other fungi (Aspergillus niveus, Aspergillus ochraceus, Aspergillus oryzae, Aspergillus terreus, Monascus ruber, Monascus purpureus and Penicillium camemberti) which are found or used in the production of human foods, such as grain, cheese, sake and red pigments. Citrinin has also been found in commercial red yeast rice supplements [3]. As one of mycotoxins, citrinin possesses antibiotic, bacteriostatic, antifungal and antiprotozoal properties. While it is also known as a hepato-nephrotoxin in a wide range of species [4-6].

Jackson and Ciegler [7] reported that heat degrades citrinin, but toxicity is retained in a fraction that is chloroform soluble and $\mathrm{NaCHO}_{3}$ insoluble. This would agree with Chu's [8] findings that antibiotic power is retained after autoclaving. Heat had caused decarboxylation of the citrinin to a compound such as decarboxycitrinin (Figure 2a), which is a natural metabolite of $P$. citrinum [2], or decarboxydihydrocitrinin (Figure 2b).

It is a quinine methide with two intramolecular hydrogen bonds [1]. Citrinin crystallizes in a disordered structure, with the p-quinone and o-quinone two tautomeric forms in a dynamic equilibrium in the solid state (Figure 3).

Yao et al. [9] found that investigation of a microbial fermentation organic extract of Penicillium sp. H9318 led to the isolation of a new isoquinolinone alkaloid, (5S)-3,4,5,7-tetramethyl-5,8-dihydroxyl$6(5 H)$ - isoquinolinone [1], along with four known citrinin derivatives [2-5] (Figure 4). Citrinin [2] exhibited significant inhibitory activity against Streptomyces 85E in the hyphae formation inhibition (HFI) assay, while compounds 1, 3-5 were not active when tested at $20 \mathrm{mg} /$ disk in the HFI assay.

\section{Cultures Conditions of Citrinin}

Citrinin was produced in liquid potato-dextrose medium (PD) as described by [10]. Inocula containing $10^{5}$ spores of each citrinin producing isolates of Penicillium citrinum were added to PD medium $(3 \mathrm{~mL})$ in $25 \mathrm{~mL}$ flasks $(20 \mathrm{x} 100 \mathrm{~mm})$ and incubated at $26 \pm 0.5^{\circ} \mathrm{C}$ for 21 days. Citrinin was extracted from cultures three times with $10 \mathrm{ml}$ of chloroform, treated with anhydrous sodium sulfate, filtered and evaporated to dryness [11]. Residues were dissolved in chloroform (0.1 $\mathrm{mL})[12]$.

Also Hajjaj et al. [13], described the production of citrinin as follows: A strain of Monascus ruber (ATCC 96218) was grown at $28^{\circ} \mathrm{C}$ in a chemically defined medium as described by. A suspension of $10^{8}$ spores was used to inoculate a 1-liter baffled Erlenmeyer flask containing $200 \mathrm{ml}$ of glucose medium. The presence of red pigments and citrinin in the culture broth was determined.<smiles>CC1=C2C(=CO[C@@H](C)[C@@H]2C)C(O)=C(C(=O)O)C1=O</smiles>

Figure 1: Structure of Citrinin
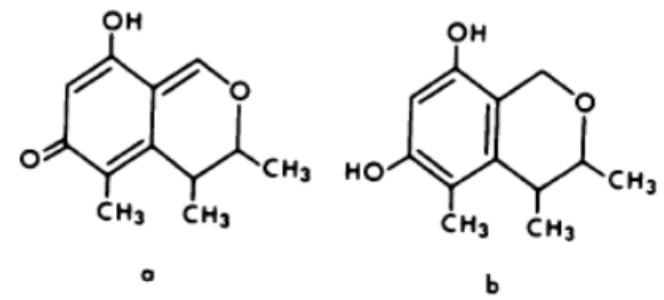

Figure 2: (a) Decarboxycitrinin; (b) decarboxydihydrocitrinin (Jackson and Ciegler 1978)
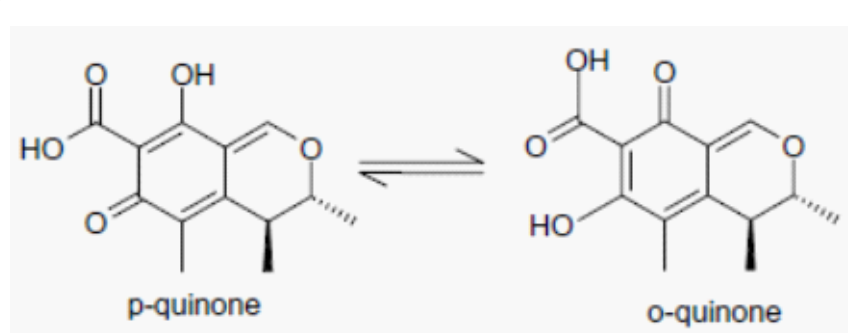

Figure 3: Structural formula of citrinin isomers [1].

*Corresponding author: Alaa Mostafa Abou-Zeid, Botany Department, Faculty of Science, Tanta University, Tanta, Egypt, E-mail: abouzeid_alaa@yahoo.com Received February 21, 2012; Accepted March 29, 2012; Published March 31, 2012

Citation: Abou-Zeid AM (2012) Review on Citrinin: Production, Effect of Some Plant Extracts and Gene Involved in its Biosynthesis. J Civil Environment Engg 2:113. doi:10.4172/2165-784X.1000113

Copyright: ( 2012 Abou-Zeid AM. This is an open-access article distributed under the terms of the Creative Commons Attribution License, which permits unrestricted use, distribution, and reproduction in any medium, provided the original author and source are credited. 


\section{Citrinin Determination}

Citrinin was isolated from the medium by filtration of the mycelium cultures on M14 membranes (pore size of $0.8 \mu \mathrm{m}$ ). The filtrate was lyophilized, re-suspended in $60 \mathrm{ml}$ of water, and extracted three times with water saturated with $n$-butanol. The organic phase was dried and vacuum concentrated, and the residue was dissolved in 50 $\mathrm{ml}$ of acidified water ( $\mathrm{pH}$ 2.0). This solution was treated twice with 120 $\mathrm{ml}$ of ethyl acetate, and the retained organic phase was extracted twice with $150 \mathrm{ml}$ of $0.4 \% \mathrm{NaHCO}_{3}$. The aqueous phase was adjusted to a $\mathrm{pH}$ of 3.0 with $\mathrm{HCl}$ and again extracted twice with $120 \mathrm{ml}$ of ethyl acetate. The organic phase containing citrinin was evaporated to dryness and resuspended in a minimal volume of water. The toxin was isolated by thin-layer chromatography in chloroform-methanol-water $(65 / 25 / 4$, $\mathrm{vol} / \mathrm{vol} / \mathrm{vol}$ ), and the band containing the toxin was solubilized in chloroform which was evaporated. Spectra were referenced internally to the solvent for ${ }^{13} \mathrm{C}$ NMR and to trimethylsilyl for ${ }^{1} \mathrm{H}$ NMR. Minimization of the relative molecular energy of intermediates 2 and 3 (Figure 5) was carried out with the molecular mechanics programs Biosym and Discover on a Silicon Graphics machine. It was found that the production of citrinin started after $45 \mathrm{~h}$ of cultivation and did not appear to stop when the glucose from the medium was consumed [14]. The citirinin concentration was $6.5 \mu \mathrm{g} / \mathrm{ml}$.

These experiments confirmed that citrinin arose from the polyketide pathway, by a route apparently similar to that found in P. citrinum and A. terreus $[15,16]$. However, they found that the spectra from labeled citrinin were strikingly different from those obtained for P. citrinum and $A$. terreus, incubated under the same conditions. The occurrence of a tetraketide as the precursonr for both citrinin and red pigments may account for the differential production of these two polyketides during the growth of M. ruber (Figure 6).

Mossini and Kemmelmeier [12] determined the citrinin as follows: reported that citrinin standard was prepared in ethanol $(1 \mathrm{mg} / \mathrm{mL})$ and stored at $4^{\circ} \mathrm{C}$ [11]. Extracts (dissolved in chloroform) and standard underwent TLC on $20 \times 20 \mathrm{~cm}$ Aluminium plates (Silica gel $60-\mathrm{G}$.), with toluene-ethyl acetate-formic acid (6:4:0.5, v/v). After development the plates were exposed to $365 \mathrm{~nm}$ ultraviolet light (UV). Citrinin appears as a fluorescent yellow spot. The phenolic group in citrinin,

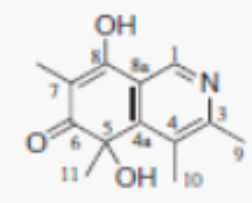

1

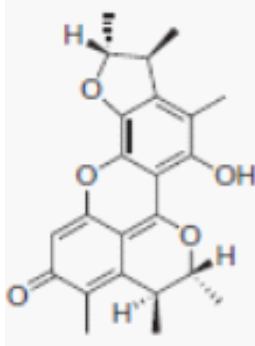

3

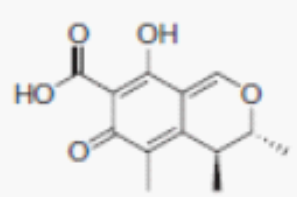

2

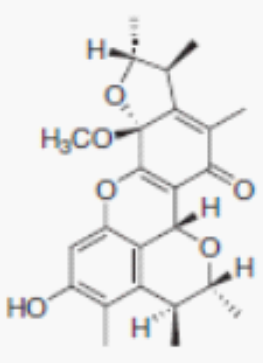

5
Figure 4: Isoquinocitrinin $A(1)$, and four known compounds, citrinin (2) penicitrone A (3), penicitrinols A (4) and B (5) [9]

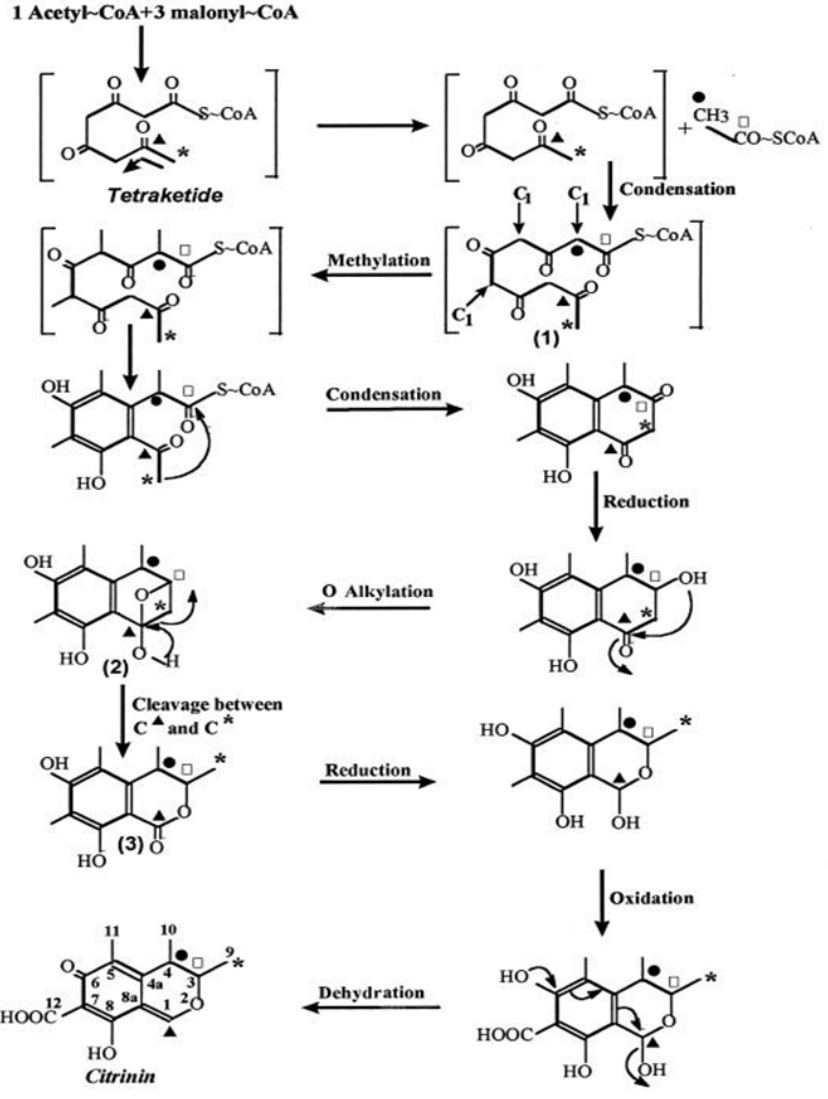

Figure 5: Scheme of the biosynthesis of citrinin by $M$. ruber. The start of the condensing reaction is indicated by the bent arrow in the upper left panel. Intermediates are numbered. Enrichment of C-1 $(\triangleleft), C-3(\Upsilon), C-9\left(^{*}\right)$, and C-4 $(\bullet)$ is indicated [14]

estimated by Folin-Phenol reagent [17] gave a linear relationship with concentration over the $5-100 \mu \mathrm{g} / \mathrm{mL}$ range. HPLC analysis was performed to confirm spectrophotometric results. Residues were dissolved in an appropriate volume of mobile phase $(1 \mathrm{~mL}$ for all samples), filtered through a $0.45 \mu \mathrm{m}$ disposable syringe filter prior to injection into the chromatograph. Aliquots $(20 \mu \mathrm{L})$ were injected on HPLC column and analyzed. Comparison of sample retention times with that of the standard identified the presence of citrinin in the samples. The relationships between peak height and area and the amount injected were linear over the ranges 2.5-50 ng. The citrinin production by the three isolates (K1, K4 and $\mathrm{k} 8$ ) of $P$. citrinum was $2.17 \times 10^{-2}, 4.25 \times 10^{-2}$ and $6.3 \times 10^{-2} \mu \mathrm{g}$ respectively as measured spectrophotometrically.

Also Reddy et al. [18] extracted and determined citrinin as follows:

The culture filtrates $(10 \mathrm{ml})$ of $P$. citrinum, isolated from rice grain, was used for extraction and estimation of citrinin. The citrinin was extracted three times with chloroform $(1: 1 \mathrm{v} / \mathrm{v})$, pooled and concentrated in vacuo at $40^{\circ} \mathrm{C}$ using a rotary evaporator. The crude extract was diluted in minimum amount of chloroform $(2 \mathrm{ml})$ and citrinin was estimated by thin layer chromatography (TLC) according to Razak et al. [19] with minor modifications. Briefly, different volumes ( 1 to $5 \mu \mathrm{l}$ ) of sample extracts were applied to precoated TLC plates (TLC Silica gel 60 F254, Merck, Germany) along with standard (containing citrinin at $0.5 \mu \mathrm{g} / \mathrm{ml}$ ). The plates were developed in toluene/ethyl acetate/formic acid $(6: 4: 0.5 \mathrm{v} / \mathrm{v})$ in glass tanks covered with aluminum 
foil [12]. After development, the plates were dried and observed under long wavelength $(365 \mathrm{~nm})$. Citrinin appears as a fluorescent yellow spot. The intensity of the sample spots was compared with that of the standard spot. The citrinin concentration was $9.2 \mu \mathrm{g} / \mathrm{ml}$.

\section{Effects of Some Plant Extracts on Citrinin Production}

\section{Neem leaf extract (NLE)}

Dried leaves of Azadirachta indica A. Juss (Meliaceae) were extracted by maceration in distilled water $(100 \mathrm{~g} / \mathrm{L})$ and stirred for five hours in the dark, at room temperature. At the end of the extraction, the material was sieved through Whatman 1 filter paper, freeze-dried, and preserved in dark flasks. A $10 \%$ aqueous extract of the residue was prepared and used [20]. Treatments in four replicates consisted of $10 \%$ freeze-dried aqueous NLE at concentrations 3.12, 6.25, 12.5, 25 and $50 \mathrm{mg} / \mathrm{mL}$ added to the PD, before autoclaving and inoculation [12]. After 21 days, quantitative determination of the extracts from liquid culture media demonstrated inhibition of citrinin production by three isolates of $P$. citrinum on media with NLE. Neem extracts of $3.12 \mathrm{mg} /$ $\mathrm{mL}$ reached $87.16 \%$ inhibition on $\mathrm{K} 4$ and $85.86 \%$ inhibition on $\mathrm{K} 1$ and $94.86 \%$ inhibition on $\mathrm{K} 8$ citrinin production at NLE $6.25 \mathrm{mg} / \mathrm{mL}$ (Figure 7).

\section{Aqueous extracts of five medicinal plants}

Leaves of five medicinal plants (Andrographis paniculata, Cymbopogon citratus, Eurycoma longifolia, Kaempferia galanga and Orthosiphon aristatus) were collected and washed under tap water. Then the leaves were dried in hot air oven at $60 \mathrm{C}$ for 4 days and ground to made powder to pass through 20 mesh sieve. Ten grams of ground powder were shaken in $100 \mathrm{ml}$ distilled water at $200 \mathrm{rpm}$ for $5 \mathrm{~h}$ at room temperature [19]. The insoluble material was filtered by Whatman No.1 filter paper and centrifuged at 10,000 rpm for $10 \mathrm{~min}$. The supernatant was collected and passed through $0.22 \mu \mathrm{m}$ membrane filter and stored at $-20^{\circ} \mathrm{C}$ until use. Various concentrations $(2.5,5.0$, 7.5 and $10.0 \mathrm{mg} / \mathrm{ml}$ ) of aqueous plant extracts were added to cooled liquid broth. A $10 \mu \mathrm{l}$ amount from suspension contained $10^{5}$ spore/ $\mathrm{ml}$ of fungus was inoculated in each flask and shaken at $200 \mathrm{rpm}$ for 10 days at $25 \pm 2^{\circ} \mathrm{C}$. The control contained PDB broth and $10 \mu \mathrm{l}$ of fungal suspension [18]. It was found that all plant extracts effectively reduced the citrinin production ranging from 42.3 to $91.3 \%$ at $10 \mathrm{mg} /$ $\mathrm{ml}$ concentration in liquid media. Among the plant extracts tested, C. citrates effectively inhibited the citrinin production by $P$. citrinum ranging from 22.8 to $91.3 \%$ followed by A. paniculata ranging from 8.6 to $83.6 \%$ at all concentrations tested. Other plant extracts showed less reduction ranging from 42.3 to $54.3 \%$ even at higher concentration (10 $\mathrm{mg} / \mathrm{ml})$.

\section{Gene Involved in Citrinin Biosynthesis}

During the biosynthesis of the secondary metabolites like citrinin, many biosynthetic enzymes are required and should function coordinately in the synthesis of these structurally complex metabolites, many of which are polyketides. Polyketides, such as pigments and mycotoxins, are structurally diverse and often complex compounds that are at least partially synthesized by multifunctional enzymes called polyketide synthases (PKSs).The genes encoding these enzymes have often been reported to localize in an adjacent region or to form a gene cluster [21], similar to the situation of biosynthetic gene clusters for secondary metabolites in prokaryotic actinomycetes.

In Shimizu et al. [22] study, a PKS gene for citrinin (CT) ( $p k s C T)$ was cloned from $M$. purpureus. They reported that PCR with primer pair KS and LC5c (Figure 8) yielded a single distinct product, and the PCR product ( $430 \mathrm{bp}$ ) was very close to the expected size, $420 \mathrm{bp}$, calculated from the sequences of other fungal PKSs. The deduced amino acid sequence was $49 \%$, similar to the aflatoxin biosynthetic PKS from A. parasiticus and $43 \%$ similar to the bikaverin biosynthetic PKS from G. fujikuroi. These similarities suggested that the PCR fragment was

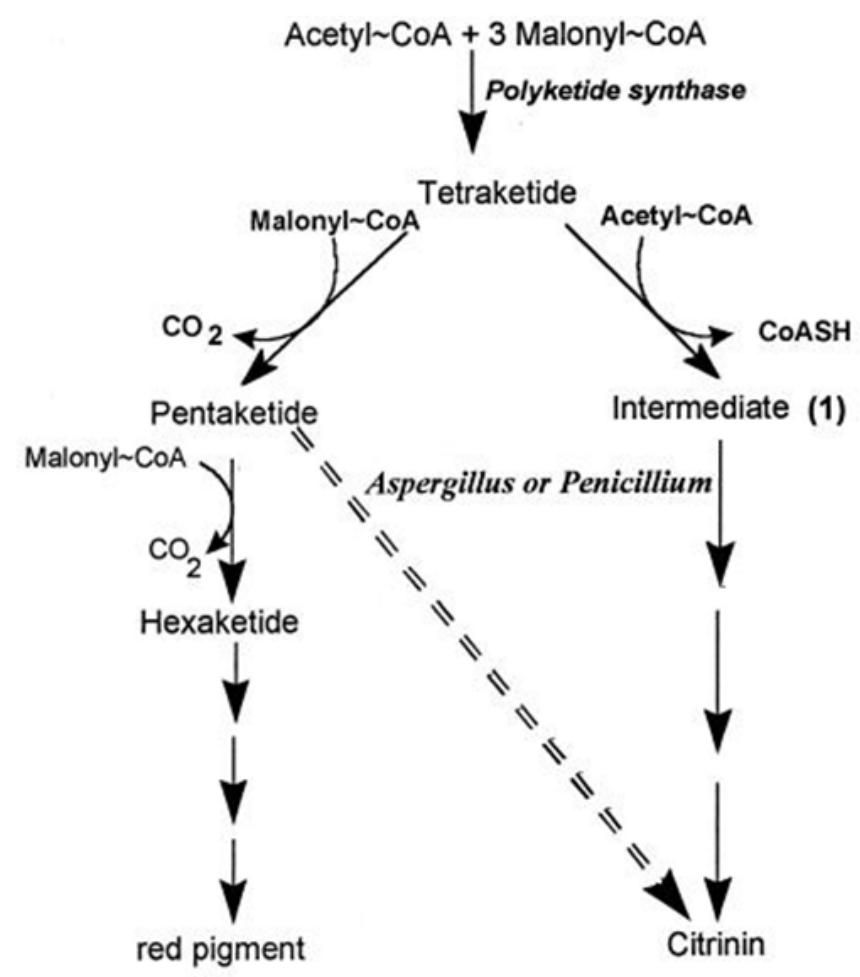

Figure 6: Biosynthesis of citrinin and red pigment in $M$. ruber. The toxin pathway in Aspergillus and Penicillium is indicated by the dashed arrow [14]

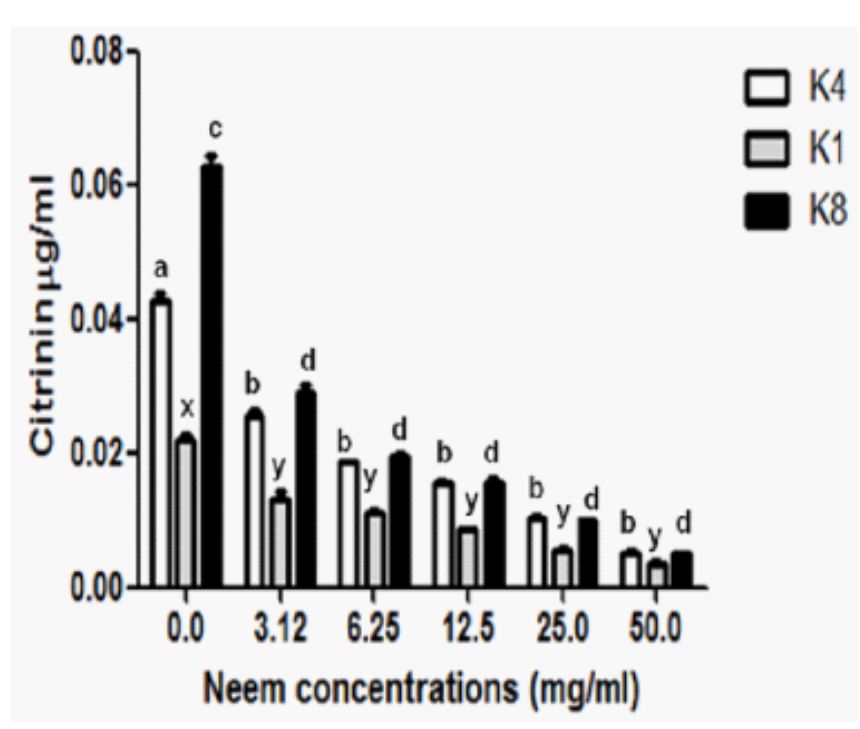

Figure 7: Citrinin production from $P$. citrinum isolates determined by spectrophotometric Assay. Bars indicate standard deviation for experiments carried out in four replicates. Different small letters over the columns indicate statistically significant differences $(p<0.05)$ for isolate $\mathrm{K} 4(\mathrm{a}, \mathrm{b}), \mathrm{K} 1(\mathrm{x}, \mathrm{y})$ and K8 (c, d) [12]. 
part of a PKS gene responsible for the biosynthesis of multi-aromaticring polyketides.

The complete PKS gene (7,838 bp; DDBJ accession no. AB167465) encoding a 2,593-amino-acid protein (Figure 9) was obtained from the $M$. purpureus genome by three rounds of colony hybridization by using probe A to identify a BamHI (1)-BamHI (2) fragment and an EcoRISalI fragment and probe B to clone a KpnI (2)-KpnI (3) fragment (Figure 9).

In Shimizu et al. [23] study, they cloned the genes in the vicinity of $p k s C T$ to obtain new genes involved in CT biosynthesis. An activator gene essential for the efficient production of CT was found in the upstream region of $p k s C T$, and they demonstrate that an extremely low-CT producer can be created by disrupting the gene.

The transcription patterns of the five ORFs were examined by RTPCR with RNA samples prepared from mycelia cultivated for 2,4 , or 6 days under CT production conditions. pksCT was transcribed from the 2-day cultivation, at which point CT production started in the wild-type strain (Figure 10A). Similarly, the transcripts of the four other plausible genes (orf2, encoding a regulator; orf 3 , encoding an oxygenase; orf4, encoding an oxidoreductase; and orf5, encoding a transporter) were detected from the 2-day cultivation, whereas no transcription of orf 1 was detected [23].

Results suggested that, as in the case of AflR in the aflatoxin biosynthetic cluster [23,24], the 2 open reading frames orf 2 product (designated CtnA for being the first factor related to CT biosynthesis other than PksCT) acts as an activator at least on pksCT and (orf5) transcription. This occurs probably through binding to specific DNA sequences in the upstream region of each of the two genes, although no

\section{Fungal PKS}

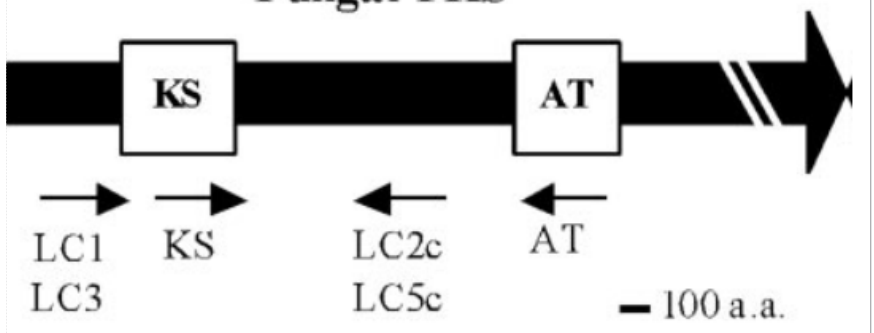

Figure 8: Primer positions in the flanking regions of KS and AT domains of a consensus fungal PKS. The boldface arrow and open boxes show the fungal PKS gene and regions encoding the KS and AT domains, respectively. The small arrows indicate the primers' positions and directions [22].

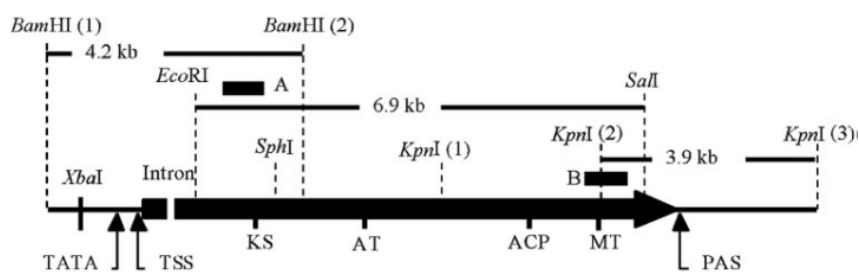

Figure 9: Restriction map of the cloned 12.9-kb BamHI (1)-Kpnl (3) fragment and structure of the cloned PKS. The black boxes indicate the locations of probes $\mathrm{A}$ and $\mathrm{B}$ used to obtain the BamHI (1)-BamHI (2) and EcoRI-Sall fragments and the Kpnl (2)-Kpnl (3) fragment, respectively, by colony hybridization. The thick black arrow indicates the deduced open reading frame for $p k s C T$ in the cloned DNA sequence. The small arrows indicate the TATA box, the transcriptional start site (TSS), and polyadenylation site (PAS), respectively [22].
(A)
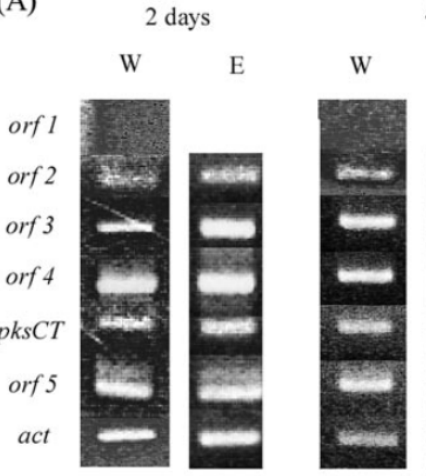

4 days

E

W

D

Figure 10: Transcriptional analysis of putative CT biosynthetic genes. (A) RTPCR was performed against RNA samples extracted from mycelia harvested from MC liquid medium after the indicated period of cultivation [23].

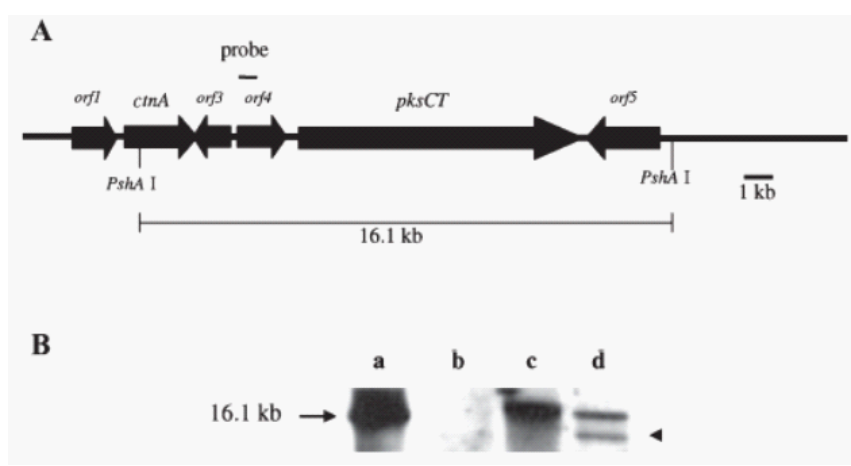

Figure 11: (A) Organization of the putative CT biosynthetic gene cluster in $\mathrm{M}$ purpureus: orfl encoding the putative dehydrogenase; ctnA encoding a positive regulator; orf3 encoding a plausible oxygenase; orf4 encoding a plausible oxidoreductase; pksCT encoding CT polyketide synthase; orf5 encoding a plausible transporter. The bar above orf4 indicates the position of the probe used in panel B. (B). Southern blot analysis of the transformants. Southern blot analysis was carried out against PshAl-digested genomic DNA using the PCR fragment (orf4-f and orf4-r primers) from orf4 as the probe. The arrow indicates the position of a 16.1-kb band corresponding to the PshAl-fragment. An arrowhead indicates the position of a 13.5-kb band corresponding to an apparently truncated cluster in lane d. Lanes: a, CossCT vector; b, A. oryzae NS4; c, strain 1-1; d, strain 16-2 [25].

definite conserved sequence was discovered in the promoter regions of pksCT and orf5.

Sakai et al. [25] found that in the region flanking pksCT, there are four more ORFs in addition to ctnA. The estimated gene products of these ORFs showed high similarity to a dehydrogenase (orf1), an oxygenase (orf3), an oxidoreductase (orf4), and a transporter (orf5), respectively (Figure 11A). Therefore, all four genes, including pksCT and ctnA, appear to constitute a CT biosynthetic gene cluster. Because the putative CT biosynthetic cluster is relatively small (around $20 \mathrm{~kb}$ ) it provided a suitable cosmid insert.

Characterization of $A$. oryzae transformants with CossCT containing the CT biosynthetic gene cluster Following a partial BamHI digestion, the fragment containing the $20-\mathrm{kb}$ CT biosynthetic cluster with sufficient flanking regions $(4 \mathrm{~kb}$ on one side and $9 \mathrm{~kb}$ on the other) was recovered from SuperCos CT-1 and was transferred into the CossC Aspergillus-E. coli shuttle vector creating CossCT. CossCT was then transformed into the heterologous host A. oryzae NS4. The transformants were selected on SC medium and their genotypes were 
confirmed by Southern blot using part of orf4 as a probe (Figure 11A and 11B). They verified that two transformants (strains 1-1 and 162 ), each contained the entire putative CT biosynthetic gene cluster, although strain 16-2 displayed an additional band with a lower molecular weight than the expected size of $16.1 \mathrm{~kb}$. This band likely arose from a truncated form of the cluster (Figure 11B).

Sakai et al. [25] concluded that introducing additional copies of an activator gene (ctnA), controlled by the Aspergillus nidulans trpC promoter, into the citrinin-cluster-containing transformants enhanced the transcription of all the genes in the cluster and resulted in an almost 400 -fold higher citrinin production compared to that of the parental transformant.

\section{References}

1. Xu B, Jia X, Gu L, Sung C (2006) Review on the qualitative and quantitative analysis of the mycotoxin citrinin. Food control 17: 271-285.

2. Deshpande SS (2002) Handbook of Food Toxicology. Marcel Dekker Inc. New York, NY, USA, 424

3. Gordon RY, Cooperman T, Obermeyer W, Becker DJ (2010) Marked Variability of Monacolin Levels in Commercial Red Yeast Rice Products: Buyer Beware. Arch Intern Med 170: 1722-1727.

4. Hanika C, Carlton WW, Tuite J (1983) Citrinin mycotoxicosis in the rabbit. Food Chem Toxicol 21: 487-493.

5. Bilgrami KS, Sinha SP, Jeswal P (1988) Nephrotoxic andhepatoxic effects of citrinin in mice (Mus musculus). Proc Indian Natn Sci Acad B54: 35-37.

6. Berndt WO (1990) Ochratoxin-citrinin as nephrotoxins. In Llewellyn GC \& Rear PCO (Edn.), Biodeterioration Research 3. New York, USA: Plenum Press $55-56$.

7. Jackson LK, Ciegler A (1978) Production and Analysis of citrinin in Corn. App Environ Microbiol 36: 408-411.

8. Chu WC (1946) Miscellaneous pharmacological actions of citrinin. J Lab Clin Med 31: $72-78$

9. Yao G, Sebisubi FM, Voo LYC, Ho CC, Tanb GT, et al. (2011) Citrinin derivatives from the soil filamentous fungus Penicillium sp. H9318. J Braz Chem Soc 22: 1125-1129.

10. Wu MT, Ayres JC, Koehler PE (1974) Production of citrinin by Penicillium viridicatum on country-cured ham. Appl Microbiol 27: 427-428.

11. Betina V (1984) Mycotoxins - Production, Isolation, Separation and Purification Elsevier Science Publishers Amsterdam Netherlands 217-235.
12. Mossini SA, Kemmelmeier C (2008) Inhibition of Citrinin Production in Penicillium citrinum Cultures by Neem [Azadirachta indica A. Juss (Meliaceae)] Int J Mol Sci 9: 1676-1684.

13. Hajjaj H, Klaebe A, Loret MO, Tzedakis T, Goma G, et al. (1997) Production and Identification of N-Glucosylrubropunctamine and N-Glucosylmonascorubramine from Monascus ruber and Occurrence of Electron Donor-Acceptor Complexes in These Red Pigments. Appl Environ Microbiol 63: 2671-2678.

14. Hajjaj H, Klaebe A, Loret MO, Goma G, Blanc PJ, et al. (1999) Biosynthetic Pathway of Citrinin in the Filamentous Fungus Monascus ruber as Revealed by ${ }^{13} \mathrm{C}$ Nuclear Magnetic Resonance. Appl Environ Microbiol 65: 311-314.

15. Barber J, Staunton J (1980) New insights into polyketide metabolism; the use of protium as a tracer in the biosynthesis of citrinin by Penicillium citrinum. J Chem Soc Perkin Trans 1: 2244-2248.

16. Sankawa U, Ebizuka Y, Noguchi H, Isikawa Y, Kitaghawa S, et al. (1983) Biosynthesis of citrinin in Aspergillus terreus. Tetrahedron 39: 3583-3591.

17. Amandioha AC (2000) Controlling rice blast in vitro and in vivo with extracts of Azadirachta indica. Crop Prot 19: 287-290.

18. Reddy KRN, Nurdijati SB, Salleh B (2010) Efficacy of aqueous medicinal plant extracts on growth and citrinin production by Penicillium citrinum isolated from rice grains. Afr J Microbiol Res 4: 2562-2565.

19. Razak MF, Aidoo KE, Candlish AG (2009) Mixed herbs drugs: Inhibitory effect on growth of the endogenous mycoflora and aflatoxin production. Mycopathologia 167: 273-286.

20. Mossini SA, de Oliveira KP, Kemmelmeier C (2004) Inhibition of patulin production by P.expansum cultured with neem (Azadirachta indica) leaf extracts. J Basic Microbiol 44: 106-113.

21. Kennedy J, Auclair K, Kendrew SG, Park C, Vederas JC, et al. (1999) Modulation of polyketide synthase activity by accessory proteins during lovastatin biosynthesis. Science 284: 1368-1372.

22. Shimizu T, Kinoshita H, Ishihara S, Sakai K, Nagai S, et al. (2005) Polyketide synthase gene responsible for citrinin biosynthesis in Monascus purpureus. Appl Environ Microbiol 71: 3453-3457.

23. Shimizu T, Kinoshita $H$, Nihira $T$ (2007) Identification and in vivo functional analysis by gene disruption of $\operatorname{ctnA}$, an activator gene involved in citrinin biosynthesis in Monascus urpureus. Appl Environ Microbiol 73: 5097-5103.

24. Ehrlich KC, Montalbano BG, Cary JW (1999) Binding of the C6-zinc cluste protein, AFLR, to the promoters of aflatoxin pathway biosynthesis genes in Aspergillus parasiticus. Gene 230: 249-257.

25. Sakai K, Kinoshita H, Shimizu T, Nihira T (2008) Construction of a citrinin gene Cluster Expression System in Heterologous Aspergillus oryzae. J Biosci Bioeng 106: 466-472. 